COLON

\title{
Do calcium channel blockers and antimuscarinics protect against perforated colonic diverticular disease? A case control study
}

\author{
C R Morris, I M Harvey, W S L Stebbings, C T M Speakman, H J Kennedy, A R Hart
}

See end of article for authors' affiliations

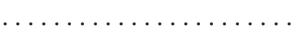

Correspondence to: C R Morris, Research Fellow, School of Medicine Health Policy and Practice, Elizabeth Fry Building, University of East Anglia, Norwich NR4 7TJ, UK; cmsword@doctors.org.uk

Accepted for publication 23 July 2003
Background: The aetiology of perforated colonic diverticular disease (PCDD) remains largely unknown. Perforation may result from a combination of high intracolonic pressures, secondary to excessive colonic segmentation, and impairment of the mucosal barrier. Calcium channel blockers and antimuscarinic drugs, which reduce colonic contractility and tone, could potentially protect against perforation. The aim of this study was to test this hypothesis using a case control design.

Methods: All cases of acute PCDD were identified over a five year period in two hospitals in Norfolk, UK. Each case was matched for age, sex, and date of admission to two controls groups: (1) patients undergoing cataract surgery and (2) patients with basal cell carcinoma. Data on drug use prior to hospital admission were obtained from medical and nursing records and compared between cases and controls. Results: A total of 120 cases of PCDD were identified and matched to 240 controls in each group. A statistically significant protective association was seen between calcium channel blocker use and PCDD using both control groups. The odds ratios were 0.41 (95\% confidence interval (Cl) $0.18-0.93$ ) using the ophthalmology control group and $0.36(95 \% \mathrm{Cl} 0.16-0.82)$ using the dermatology control group.

Conclusions: This study has shown for the first time that a protective association exists between calcium channel blockers and PCDD. The validity of this association is supported by the consistent finding in both control groups and the plausible biological mechanisms. Further studies are required to confirm this association but calcium channel blockers may represent a potential preventive therapy in PCDD.
$\mathrm{P}$ erforation of a colonic diverticulum is a serious condition with case fatality rates of between $12 \%$ and $36 \% .{ }^{12}$ The illness also results in considerable morbidity with many patients requiring multiple operations and the formation of a stoma. In the UK, it has been estimated that approximately 2000 people each year will suffer from an abscess or generalised peritonitis secondary to diverticular perforation. ${ }^{3}$ The incidence of these severe complications rises with age ${ }^{3}$ so that an increasing number of people are likely to be affected in the future, requiring substantial extra resources from healthcare services. Currently, the aetiology of diverticular perforation is largely unknown and therefore prevention is impossible.

Two factors are likely to be important in the mechanism of diverticular perforation. Firstly, excessive colonic segmentation may increase intracolonic pressures leading to mechanical weakening of the thin diverticular wall. ${ }^{4-6}$ Secondly, impairment of the mucosal barrier of the diverticulum, through alterations in mucus secretion, microbial colonisation, or epithelial cell function, ${ }^{7}$ may lead to further weakening. Therefore, drugs that reduce intracolonic pressures or protect the colonic mucosa may help to prevent perforation. To date, the only medications found to be associated with diverticular perforation are non-steroidal anti-inflammatory drugs (NSAIDs) ${ }^{8-10}$ which may cause this complication by impairing the integrity of the mucosa. ${ }^{11}$

Calcium channel blockers are a group of drugs that could potentially protect against perforation. They relax gastrointestinal smooth muscle and reduce the frequency of high pressure waves in the colon. ${ }^{12}$ Furthermore, nifedipine has been shown to increase gastrointestinal mucosal blood flow and protect against immunosuppressant induced intestinal injury. ${ }^{13}$ Although calcium channel blockers are most commonly prescribed for ischaemic heart disease and hypertension, their smooth muscle relaxant properties have been used to treat patients with irritable bowel syndrome, ${ }^{15}$ oesophageal spasm, ${ }^{16}$ and anal fissures. ${ }^{17}$ However, no study has examined their role in the treatment of colonic diverticular disease or in preventing perforation. A second group of drugs that might protect against perforation are those with antimuscarinic properties which reduce gastrointestinal tone ${ }^{18}$ and eliminate the prolonged postprandial colonic activity seen in diverticular disease. ${ }^{19}$ Antimuscarinic medications are commonly prescribed for depression, psychoses, Parkinson's disease, bladder instability, and respiratory conditions. Their gastrointestinal relaxant properties have also been used to treat patients with irritable bowel syndrome but no study has investigated whether they may protect against diverticular perforation. The aim of this case control study was to test, for the first time in an epidemiological study, the hypotheses that both calcium channel blockers and antimuscarinic drugs protect against perforated colonic diverticular disease (PCDD).

\section{METHODS}

Identification and confirmation of case group

Hospital inpatient data were used to identify patients with PCDD admitted to two hospitals, which serve a population of approximately 750000 people (Norfolk and Norwich University Hospital NHS Trust, Norwich and James Paget Hospital, Great Yarmouth, Norfolk, UK). A computer search of the international classification of disease (ICD-10) codes was used to identify patients admitted to hospital between

Abbreviations: PCDD, perforated colonic diverticular disease; NSAIDs, non-steroidal anti-inflammatory drugs 
1 April 1995 and 27 February 2000. These codes were K57.2 (perforation, abscess, or peritonitis of large intestinal diverticula); K57.4 (perforation, abscess, peritonitis, of both the small and large bowel diverticula); and K57.8 (perforation, abscess, or peritonitis of either the small or large bowel diverticula).

The hospital records of all potential cases were reviewed by the principal investigator (CRM) to confirm the diagnoses. A patient was included in the case group if there was evidence of a macroscopic colonic diverticular perforation at either laparotomy or post mortem. In the absence of a macroscopic perforation, patients were included if there was evidence of extracolonic pus or faeces, in the presence of an inflamed diverticulum, and with no other intra-abdominal pathology (implying a sealed perforation). Finally, patients who did not undergo laparotomy were included if there was radiological evidence (computed tomography, ultrasound, or contrast enema) of a colonic diverticular abscess. Patients with the chronic sequelae of perforation such as colonic stricture and fistulae were excluded. These complications may be present for some time before admission to hospital, presenting difficulties in determining whether drug exposures occurred before the onset of the illness.

\section{Selection of the control groups}

Two control groups were used, with each case matched for age (within one year), sex, and hospital of admission to two patients from each control group. The first control group were patients admitted for cataract surgery within three months of the case admission (ophthalmology control group). The second control group were outpatients who had attended for excision of non-melanotic skin tumours within three months of the case admission (dermatology control group). These two control groups were chosen because patients with these diagnoses were likely to use calcium channel blockers and antimuscarinic drugs with similar rates to that of the general population and were likely to be within a similar age range as the case group.

\section{Measurement of exposures}

Data on medication use was obtained for both cases and controls from the general practitioner's referral letter and medical and nursing records. The primary exposure measures were oral intake of calcium channel blockers and antimuscarinic drugs immediately prior to the onset of symptoms of perforation. For calcium channel blockers, individual drugs were classed as either short acting or modified release preparations depending on their dosage, frequency of use, and classification in the British National Formulary. For antimuscarinic drugs, only those classed as having major systemic effects were included. These were tricyclic antidepressants, phenothiazines, gastrointestinal smooth muscle relaxants, drugs for bladder instability, and antiparkinsonian drugs. To assess whether group differences in the use of calcium channel blockers simply related to differences in comorbidity and overall drug use, data were also recorded on the number of medications used by each patient, the use of all cardiovascular medications, and the prevalence of cardiovascular comorbidity.

\section{Analysis}

All data were anonymised, coded, and entered into the database of the statistics program STATA 5.0 for Windows 95 (Stata Corporation, Texas, USA). Two separate analyses were performed comparing the prevalence of drug use between the case group and each of the control groups. Conditional logistic regression was used to calculate odds ratios with $95 \%$ confidence intervals $(95 \% \mathrm{CI})$, representing the risk of developing a perforation if taking a drug compared with not taking that drug. These odds ratios were adjusted for the confounding effect of NSAID medication, the only known risk factor for this condition. Primary analyses were undertaken for all calcium channel blockers and antimuscrainics. Further analyses were performed for short acting and modified release calcium channel blockers and tricyclic antidepressants, which accounted for the majority of antimuscarinics. The use of cardiovascular medication and prevalence of cardiovascular comorbidity were also compared using conditional logistic regression. Differences in the number of medications taken by cases and controls were compared using the Wilcoxon signed rank test.

\section{RESULTS}

\section{Case group characteristics}

A total of 120 consecutive patients with confirmed PCDD were identified during the study period. The case group consisted of $39(32.5 \%)$ men and 81 (67.5\%) women with a median age of 74 years (range 30-95). Prior to hospital admission, 46 people $(38 \%)$ were known to have colonic diverticular disease from previous radiological or endoscopic investigations. However, only 16 (13\%) had previously been admitted to hospital with a complication of diverticular disease. The clinical management was surgical in 107 (89\%) cases, the most frequent operative finding being purulent peritonitis $(43 \%)$ followed by abscess $(23 \%)$ and faecal peritonitis $(22 \%)$. The overall mortality rate was $26 \%$, ranging from $14 \%$ in patients with an abscess to $48 \%$ in those with faecal peritonitis. It was possible to accurately match case group patients so that the age and sex characteristics of cases and controls were identical.

\section{Use of calcium channel blockers}

Calcium channel blockers were used by only $6.7 \%(n=8)$ of patients in the case group compared with $14.2 \%(\mathrm{n}=34$, $\mathrm{p}=0.03)$ of the ophthalmology control group and $15.8 \%$ $(\mathrm{n}=38, \mathrm{p}=0.01)$ of the dermatology group. The specific drugs used by patients in the case group were nifedipine $(n=3)$, amlodipine $(n=2)$, verapamil $(n=2)$, and diltiazem $(\mathrm{n}=1)$. In the ophthalmology control group, nifedipine $(n=13)$ and diltiazem $(n=14)$ were most frequently used, while in the dermatology group nifedipine $(n=18)$ and amlodipine $(\mathrm{n}=12)$ were most commonly consumed. Overall use of calcium channel blockers showed a strong protective association against diverticular perforation in both control group analyses (table 1). All of this association appeared attributable to the use of modified release preparations. Adjustment for the use of NSAIDs had no effect on these protective odds ratios.

\section{Use of antimuscarinic drugs}

Antimuscarinic drugs were used by $11.7 \% \quad(n=14)$ of patients in the case group compared with 9.2\% $(\mathrm{n}=22)$ in the ophthalmology group and $10.0 \% \quad(n=24)$ in the dermatology group. These differences were not statistically significant (table 2 ). The most commonly prescribed drugs with antimuscarinic properties were tricyclic antidepressants and although these were more frequently used by control group patients, this difference was not statistically significant (table 2).

\section{Overall drug use and comorbidity}

Although calcium channel blockers were more commonly used by patients in the control groups compared with the case group, there were no differences in the use of all types of cardiovascular medication (case group $41 \%$, ophthalmology control group 44\%, and dermatology control group 46\%). Rates of cardiovascular disease were also similar (case group $33 \%$, ophthalmology group $38 \%$, and dermatology group 
Table 1 Comparison of the use of calcium channel blockers between patients with colonic diverticular perforation and two hospital based control groups

\begin{tabular}{|c|c|c|c|c|c|}
\hline \multirow[b]{2}{*}{ Drug } & \multirow{2}{*}{$\begin{array}{l}\text { Patients with PCDD } \\
(n=120) \\
(\% \text { use) }\end{array}$} & \multicolumn{2}{|c|}{$\begin{array}{l}\text { Ophthalmology controls } \\
(\mathrm{n}=240)\end{array}$} & \multicolumn{2}{|c|}{$\begin{array}{l}\text { Dermatology controls } \\
(n=240)\end{array}$} \\
\hline & & $\%$ use & OR $(95 \% \mathrm{Cl})^{*}$ & $\%$ use & OR $(95 \% \mathrm{Cl})^{*}$ \\
\hline $\begin{array}{l}\text { All calcium channel } \\
\text { blockers }\end{array}$ & 6.7 & 14.2 & $0.4(0.2-0.9)$ & 15.8 & $0.4(0.2-0.8)$ \\
\hline $\begin{array}{l}\text { Modified release } \\
\text { preparations }\end{array}$ & 25 & 83 & $0.3(0.1-0.9)$ & 117 & $02101-061$ \\
\hline Short acting preparations & 4.2 & 5.9 & $0.7(0.2-2.0)$ & 4.6 & $0.9(0.3-2.7)$ \\
\hline
\end{tabular}

$36 \%$ ). The number of drugs (all types) taken per patient was higher in the case group (median = 3) than in both the ophthalmology control group (median $=2, \mathrm{p}<0.01$ ) and the dermatology control group (median $=1, \mathrm{p}<0.01$ ).

\section{DISCUSSION}

This case control study has shown for the first time that a protective association exists between calcium channel blocking drugs and PCDD. This association appears to be solely attributable to the modified release preparations of these drugs. No protective effect against perforation was found for antimuscarinic drugs.

The main potential source of bias in this study is likely to arise from inaccuracies in the recording of drug use in hospital records. Bias could arise if the completeness of medication histories differed between cases and controls. The increased use of calcium channel blockers in the control groups might reflect a more thorough drug history or a better recall of medication in a group of healthier patients. However, this explanation is unlikely as patients admitted with perforation were recorded as taking more medications than either of the two control groups and the prevalence of cardiovascular medication use was similar for all groups (41$46 \%$ ). These rates are also similar to the reported prevalence of cardiovascular medication use in a large UK population survey of older people $(38-47 \%) .^{20}$ This suggests that the hospital records were accurate for the recording of cardiovascular medication for both cases and controls. Furthermore, the similarity of cardiovascular drug use between the control groups in this study and the UK population survey ${ }^{20}$ suggests that these were valid groups to use.

Selection bias is unlikely to have influenced the findings of this study as all patients with a diverticular perforation who were eligible for inclusion in this study should have been identified. Patients with an abscess or peritonitis secondary to diverticular perforation nearly always require hospital admission. Furthermore, the ICD-10 codes used in this investigation were shown to have a high sensitivity for identifying cases of diverticular perforation in a previous study. ${ }^{3}$ Finally, the selection criteria included only the severe manifestations of diverticular perforation which are diagnoses easily confirmed by reviewing hospital records. Consequently, the selection criteria were not open to diagnostic interpretation, minimising the chance of misclassification.

The use of hospital control groups can be problematic but in this study we used two different groups of patients with conditions that have no known link with the use of calcium channel blockers. All patients with a previous history of complicated diverticular disease were excluded from the control groups but the exact prevalence of asymptomatic or mildly symptomatic diverticular disease in these groups was unknown. Previous studies however have suggested that in a population with a median age of 74 years (as in the control groups), the prevalence of diverticular disease will be as high as $65 \%{ }^{21}$ This supports the conclusion that the protective effect of calcium channel blockers is associated with the perforation of a diverticulum rather than its initial formation.

The consistency of the findings between cases and both control groups greatly strengthens the observation that calcium channel blocker use is lower in patients with colonic diverticular perforation. Furthermore, the strength of the protective effect of calcium channel blockers and plausible biological mechanisms for this effect suggests that the association is real. Calcium channels are involved in the generation of myoelectrical activity and smooth muscle contraction throughout the colon. L-type calcium channel blockers, which include most of the drugs in clinical use, selectively reduce the amplitude and duration of slow wave action potentials generated by colonic pacemaker cells without affecting their frequency. ${ }^{22}$ This may produce a beneficial reduction in the strength and duration of colonic contractions, minimising episodes of high intracolonic pressure while maintaining basal activity and colonic transit. Clinically, calcium channel blockers have been shown to suppress the colonic pressure waves normally associated with eating $^{12}$ and parasympathetic stimulation, ${ }^{23}$ particularly in

Table 2 Comparison of the use of antimuscarinic drugs between patients with colonic diverticular perforation and two hospital based control groups

\begin{tabular}{|c|c|c|c|c|c|}
\hline \multirow[b]{2}{*}{ Drug } & \multirow{2}{*}{$\begin{array}{l}\text { Patients with PCDD } \\
(n=120) \\
(\% \text { use })\end{array}$} & \multicolumn{2}{|c|}{$\begin{array}{l}\text { Ophthalmology controls } \\
\text { ( } n=240)\end{array}$} & \multicolumn{2}{|c|}{$\begin{array}{l}\text { Dermatology controls } \\
(n=240)\end{array}$} \\
\hline & & $\%$ use & OR $(95 \% \mathrm{Cl})^{*}$ & $\%$ use & OR $(95 \% \mathrm{Cl})^{*}$ \\
\hline $\begin{array}{l}\text { All antimuscarinics } \\
\text { Tricyclic antidepressants }\end{array}$ & $\begin{array}{r}11.7 \\
3.3\end{array}$ & $\begin{array}{l}9.2 \\
5.0\end{array}$ & $\begin{array}{l}1.3(0.6-2.8) \\
0.7(0.2-2.1)\end{array}$ & $\begin{array}{r}10.0 \\
7.1\end{array}$ & $\begin{array}{l}1.2(0.6-2.4) \\
0.5(0.2-1.4)\end{array}$ \\
\hline
\end{tabular}

PCDD, perforated colonic diverticular disease.

*Odds ratio (OR) (with $95 \%$ confidence interval (CI)) of developing PCDD if taking a drug compared with not taking a drug. 
patients with excessive colonic contractility. ${ }^{24} 25$ Antimuscarinic drugs have similar effects in blocking extrinsic stimuli but do not affect slow wave activity. ${ }^{19}$ The lack of a protective effect for antimuscarinics may indicate that suppression of slow wave amplitude and duration is important in protecting against perforation. Alternatively, calcium channel blockers may be acting through other mechanisms such as increasing gastrointestinal mucosal blood flow, helping to promote cytoprotective activity and repair in the diverticular mucosa. ${ }^{14} \mathrm{~A}$ further possibility is that the duration of action of a drug is important as the protective association of calcium channel blockers was attributable to modified release drugs. Modified release preparations are likely to produce more gradual and sustained effects on motility, which may explain why no association was seen for shorter acting calcium channel blockers and antimuscarinics.

In view of the findings of this investigation, further aetiological studies are required to confirm the protective association between calcium channel blockers and perforated colonic diverticular disease. These studies should ideally involve community control groups and use interviews with patients to obtain more detailed data, particularly on the duration of use of medications. Confirmation of a causal relationship would support therapeutic trials of calcium channel blockers in patients at high risk of developing complications secondary to diverticular perforation. Such a group might include those who have had two or more episodes of inflammation and who would currently be advised to undergo surgical resection. This study has also shed light on possible mechanisms through which diverticular perforation may be prevented. Future investigations should examine other pharmacological factors that reduce colonic motility or augment mucosal blood flow. Identification of an effective drug treatment for preventing perforation would be a major advance in the management of patients with known colonic diverticular disease. As well as preventing perforation, drugs such as calcium channel blockers might also help to reduce the abdominal symptoms attributed to colonic spasm. Such a measure could potentially improve the quality of life of patients as well as reducing the healthcare resources required to treat them.

\section{ACKNOWLEDGEMENTS}

Funding for this study was from the Directorate of Health and Social Care, Department of Health, UK, and the Norfolk and Norwich Hospitals Bicentenary Trust.

We would like to acknowledge the help and cooperation of staff in the departments of clinical coding and medical records as well as all consultant general surgeons, ophthalmologists, dermatologists, and plastic surgeons at the Norfolk and Norwich University Hospital NHS Trust and the James Paget Hospital NHS Trust.

\section{Authors' affiliations}

C R Morris, School of Medicine Health Policy and Practice, University of East Anglia, Norwich, UK

I M Harvey, School of Medicine Health Policy and Practice, University of East Anglia, Norfolk and Norwich University Hospital NHS Trust, Norwich, UK

W S L Stebbings, C T M Speakman, Department of General Surgery,
Norfolk and Norwich University Hospital NHS Trust, Norwich, UK H J Kennedy, Department of Gastroenterology, Norfolk and Norwich University Hospital NHS Trust, Norwich, UK

A R Hart, School of Medicine Health Policy and Practice, University of East Anglia, and Department of Gastroenterology, Norfolk and Norwich University Hospital NHS Trust, Norwich, UK

\section{REFERENCES}

1 Elliott TB, Yego S, Irvin TT. Five-year audit of the acute complications of diverticular disease. Br J Surg 1997:84:535-9.

2 Tudor RG, Farmakis N, Keighley MR. National audit of complicated diverticular disease: analysis of index cases. Br J Surg 1994;81:730-2.

3 Hart AR, Kennedy HJ, Stebbings WS, et al. How frequently do large bowel diverticula perforate? An incidence and cross-sectional study. Eur J Gastroenterol Hepatol 2000;12:661-5.

4 Arfwidsson S, Kock NG, Lehmann L, et al. Intraluminal pressure in the sigmoid colon of normal subjects and patients with diverticular disease. Acta Chir Scand 1964:342:1-132.

5 Painter NS, Truelove SC. The intraluminal pressure patterns in diverticulosis of the colon. Part II The effect of morphine. Gut 1964;5:207-13.

6 Watters DA, Smith AN. Strength of the colon wall in diverticular disease. Br J Surg 1990;77:257-9.

7 Edwards C. Physiology of the colorectal barrier. Adv Drug Delivery Rev 1996;28:173-90.

8 Wilson RG, Smith AN, Macintyre IM. Complications of diverticular disease and non-steroidal anti-inflammatory drugs: a prospective study. $\mathrm{Br} J$ Surg 1990;77:1103-4.

9 Campbell K, Steele RJ. Non-steroidal anti-inflammatory drugs and complicated diverticular disease: a case-control study. Br J Surg 1991;78:190-1.

10 Corder A. Steroids, non-steroidal anti-inflammatory drugs, and serious septic complications of diverticular disease. BMJ 1987;295:1238.

11 Davies NM. Toxicity of nonsteroidal anti-inflammatory drugs in the large intestine. Dis Colon Rectum 1995;38:1311-21.

12 Bassotti G, Calcara C, Annese V, et al. Nifedipine and verapamil inhibit the sigmoid colon myoelectric response to eating in healthy volunteers. Dis Colon Rectum 1998;41:377-80.

13 Greenstein SM, Sun S, Kim DY, et al. Nifedipine prevents tacrolimus-induced intestinal hemodynamic and functional impairments. Transplant Proc 1998:30:2668-9.

14 Sun S, Greenstein SM, Kim DY, et al. Nifedipine protects small intestine from cyclosporine-induced hemodynamic and functional impairment. J Surg Res 1997;69:295-9.

15 Scarpignato C, Pelosini I. Management of irritable bowel syndrome: novel approaches to the pharmacology of gut motility. Can J Gastroenterol 1999; 13(suppl A):50-65A.

16 Short TP, Thomas E. An overview of the role of calcium antagonists in the treatment of achalasia and diffuse oesophageal spasm. Drugs 1992:43:177-84.

17 Cook TA, Humphreys MM, McC Mortensen NJ. Oral nifedipine reduces resting anal pressure and heals chronic anal fissure. $\mathrm{Br} J$ Surg 1999;86:1269-73.

18 O'Brien MD, Camilleri M, Thomforde GM, et al. Effect of cholecystokinin octapeptide and atropine on human colonic motility, tone, and transit. Dig Dis Sci 1997:42:26-33.

19 Suchowiecky M, Clarke DD, Bhasker M, et al. Effect of secoverine on colonic myoelectric activity in diverticular disease of the colon. Dig Dis Sci 1987;32:833-40

20 Chen YF, Dewey ME, Avery AJ. Self-reported medication use for older people in England and Wales. J Clin Pharm Ther 2001;26:129-40.

21 Painter NS, Burkitt DP. Diverticular disease of the colon, a 20th century problem. Clin Gastroenterol 1975:4:3-21.

22 De Ponti F, Giaroni C, Cosentino M et al. Calcium-channel blockers and gastrointestinal motility: basic and clinical aspects. Pharmacol Ther 1993;60: 121-48

23 Passaretti S, Sorghi M, Colombo E, et al. Motor effects of locally administered pinaverium bromide in the sigmoid tract of patients with irritable bowel syndrome. Int J Clin Pharmacol Ther Toxicol 1989:27:47-50.

24 Awad RA, Cordova VH, Dibildox M, et al. Reduction of post-prandial motility by pinaverium bromide a calcium channel blocker acting selectively on the gastrointestinal tract in patients with irritable bowel syndrome. Acta Gastroenterol Latinoam 1997;27:247-51.

25 Sun WM, Edwards CA, Prior A, et al. Effect of oral nicardipine on anorectal function in normal human volunteers and patients with irritable bowel syndrome. Dig Dis Sci 1990;35:885-90. 